\title{
Two-Stage BJT Amplifier with Negative Voltage Series Feedback Analysis Program Proteus 8
}

\author{
San Thiri Aung ${ }^{1}$, Kaythi Wut Mhone Khin ${ }^{2}$ and Thae Su Lwin ${ }^{3}$ \\ Assistant Lecturer ${ }^{1}$ Lecturer $^{2}$ and Tutor $^{3}$ \\ ${ }^{123}$ Faculty of Computer Systems and Technologies \\ University of Computer Studies (Mandalay) \\ Mandalay, Mandalay Division
}

Myanmar

\begin{abstract}
In this paper, the common two stage amplifier voltage series negative feedback amplifier circuit is analyzed theoretically. With negative feedback, the closed-loop voltage gain can be reduced and controlled so that the op-amp can function as a linear amplifier. Negative feedback is the basic method and essential means of improving amplifying circuit efficiency. Then using Proteus 8 professional as simulation software, the relationship between the gain obtained by open-loop and the simulation of the closed loop and the pass-band is shown by comparing the real experimental data with the result of the circuit's simulation and the waveform. Negative feedback opposes or subtracts from the input signals giving it many advantages in the design and stabilization of control systems. Basically the relationship consistent with the calculated results, that is in good agreement with the theoretical analysis.
\end{abstract}

Key Words: Voltage Series Negative Feedback Amplifier, Proteus 8 Professional Software, Common-Emitter Amplifier.

\section{INTRODUCTION}

Negative (Inverse) Feedback or degenerative when the feedback signal is out of phase with the input signal and subtracts from it. This results in reduction in the gain of the amplifier. Feedback is the process of picking up a part of the output signal of an amplifier and feeding it at the input. The voltage so fed back to the input is called the voltage feedback. In voltage feedback, the feedback voltage is proportional to the output voltage. The feedback loop makes use of active linear feedback network. The following primarily looks at negative feedback amplifier circuit of the AC voltage series. The shared testing is carried out via the actual effects of the experimental data and the simulation data results.

Negative feedback opposes or subtracts from the input signals giving it many advantages in the design and stabilization of control systems. For example, if the systems output changes for any reason, then negative feedback affects the input in such a way as to counteract the change. Negative feedback is the basic method and essential means of improving amplifying circuit efficiency. The negative amplification circuit is actually a large part of the real amplification [1]. Negative feedback can increase amplifier efficiency, decrease nonlinear distortion and expand amplifier pass band.

\section{NEGATIVE FEEDBACK SYSTEM}

Negative Feedback is the most common form of feedback control configuration used in process, micro-computer and amplification systems. Operational amplifiers (op-amps) are the most commonly used type of linear integrated circuit but they have a very high gain. Basic Ideal amplifier: this provides gain A to the signal Xi fed to it as shown in figure 1 .

Sampling Network: It samples the output signal X0 and applies this signal to the feedback network. Sampling may be of two types (a) Voltage sampling and (b) Current sampling shown respectively in Figure 2. Feedback network is generally in the form of passive two port network and may be formed of resistor, capacitors, and inductors. In most cases, however, the feedback network uses only resistor [6]. 
Mixing Network: The voltage at the output of feedback network is mixed with the signal voltage in one of the following two ways (i) Series mixing and (ii) shunt mixing as shown in Figure 2.

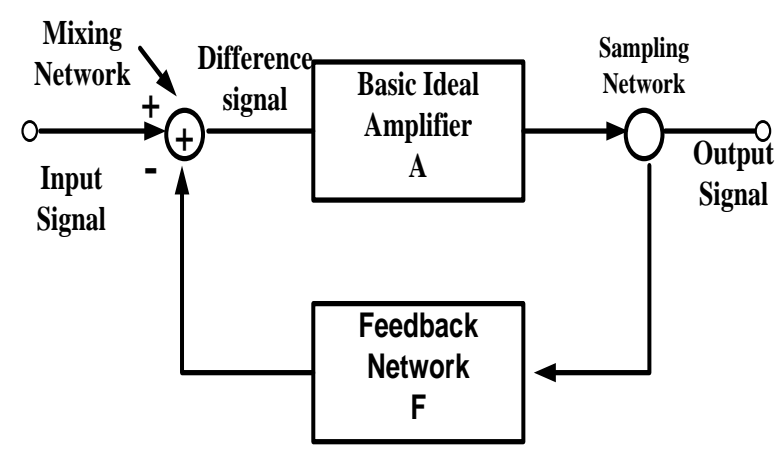

Figure 1. Block Diagram of an Idea Single Loop Feedback Amplifier
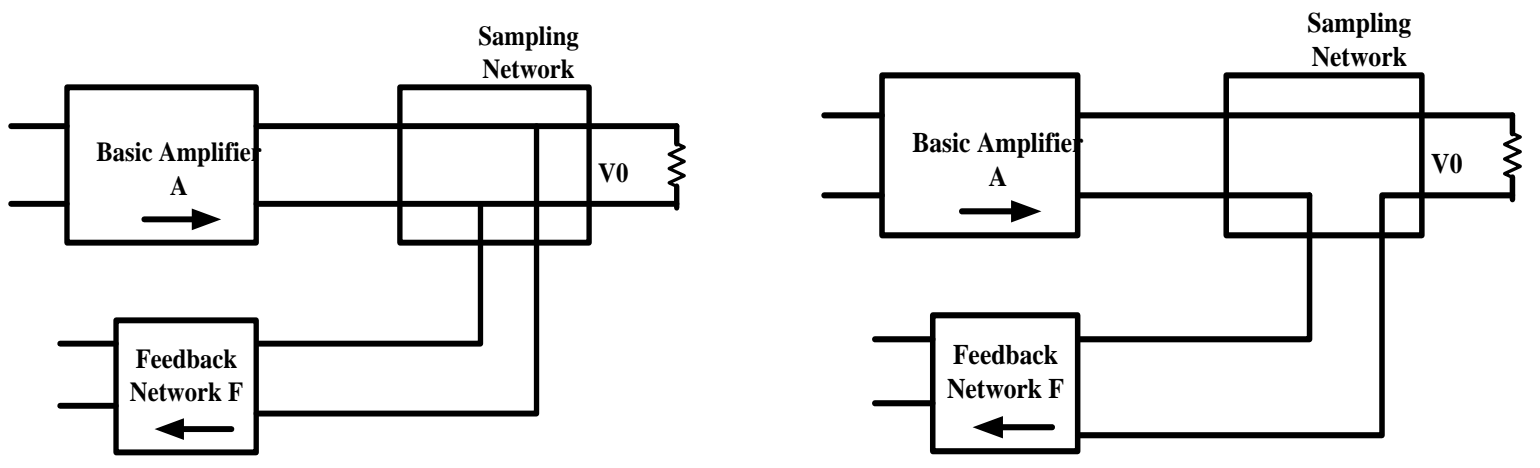

Figure 2. Output Voltage and Current Sampling in a feedback Amplifier

\section{TWO STAGE CE AMPLIFIER USING SERIES VOLTAGE NEGATIVE FEEDBACK}

A two stage, capacitor coupled BJT amplifier is shown in Figure 3. This is the same two stage CE Amplifier circuit discussed already with the addition of feedback components $R_{F 1}, R_{F 2}$. The output voltage is divided across $R_{F 2}$, and $R_{F 1}$, to produce a feedback voltage in series with the signal at the base of Q1. From previous topics of a Two Stage CE Amplifier [7], it is known that the circuit output voltage is in phase with the signal voltage. When the instantaneous level of $\mathrm{v}_{\mathrm{s}}$ is positive-going, $\mathrm{v}_{\mathrm{o}}$ is also positive-going, and consequently $\mathrm{v}_{\mathrm{f}}$ is in phase-opposition with $\mathrm{v}_{\mathrm{s}}$. When $\mathrm{v}_{\mathrm{s}}$ goes up (positively), $\mathrm{v}_{\mathrm{f}}$ also goes up, and consequently, the voltage $\left(\mathrm{v}_{\mathrm{i}}\right)$ at the base-emitter of $\mathrm{Q}_{1}$ is reduced from $\mathrm{v}_{\mathrm{s}}$ to. The feedback factors for the circuit are shown in Figure 4.

The circuit input impedance is increased by negative feedback, so input coupling capacitor $\mathrm{C}_{1}$ can be recalculated as a smaller capacitor, $\left(\mathrm{X}_{\mathrm{C} 1}=\mathrm{Z}_{\text {in }} / 10\right.$ at $\left.\mathrm{f}_{1}\right)$. Recall that the bias resistors are not affected by feedback. Regardless of how high the circuit input impedance might become, $\mathrm{C}_{1}$ should normally be selected as a minimum of $0.1 \mu \mathrm{F}$, to ensure that it is much larger than any stray capacitance at the circuit input [4].

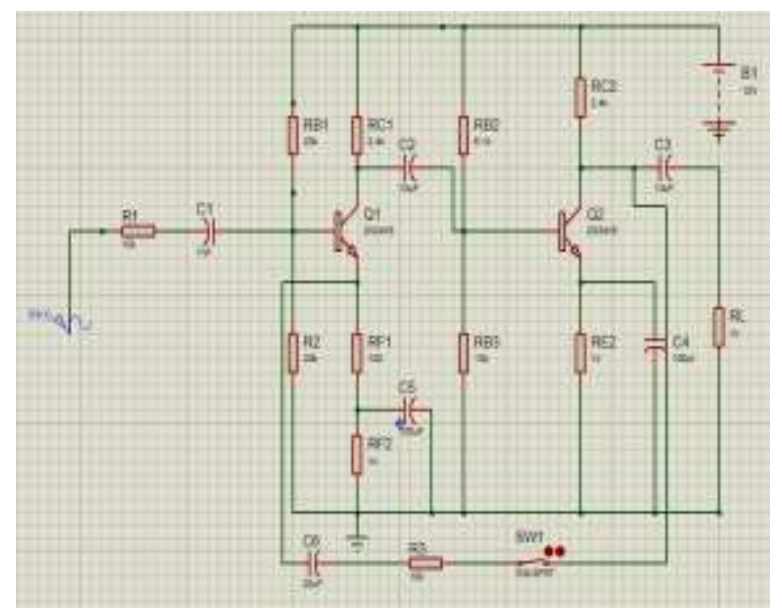

Figure 3. Negative feedback using BJT Amplifier 
International Journal of Advances in Scientific Research and Engineering (ijasre), Vol 6 (5), May-2020
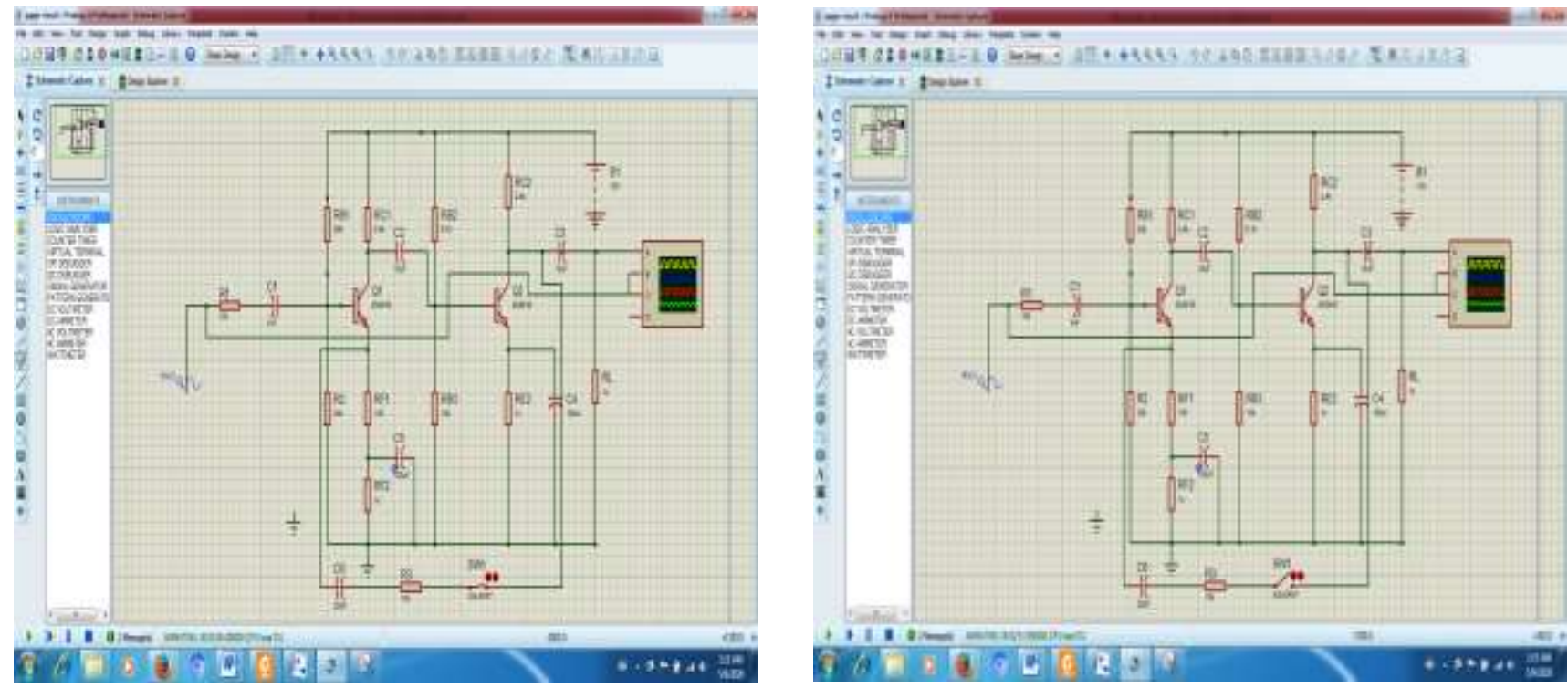

Figure 4. With and Without Negative feedback Amplifier Circuit

\subsection{Calculation and Analysis of Proteus 8 Simulation Result}

Table 1.Calculation and analysis of Proteus 8 simulation result

\begin{tabular}{|c|l|l|l|l|}
\hline Actual measure & VB & \multicolumn{1}{|c|}{ VE } & \multicolumn{1}{|c|}{ VC } & \multicolumn{1}{c|}{ IC } \\
\hline First Stage & $2.9 \mathrm{~V}$ & $2.5 \mathrm{~V}$ & $7.08 \mathrm{~V}$ & $1.98 \mathrm{~mA}$ \\
\hline Second Stage & $2.64 \mathrm{~V}$ & $1.98 \mathrm{~V}$ & $7.41 \mathrm{~V}$ & $2.11 \mathrm{~mA}$ \\
\hline Simulation measure & VB & VE & VC & IC \\
\hline First Stage & 2.77 & 1.99 & $6.94 \mathrm{~V}$ & $2.33 \mathrm{~mA}$ \\
\hline Second Stage & 2.9 & 1.88 & $7.29 \mathrm{~V}$ & $1.9 \mathrm{~mA}$ \\
\hline
\end{tabular}
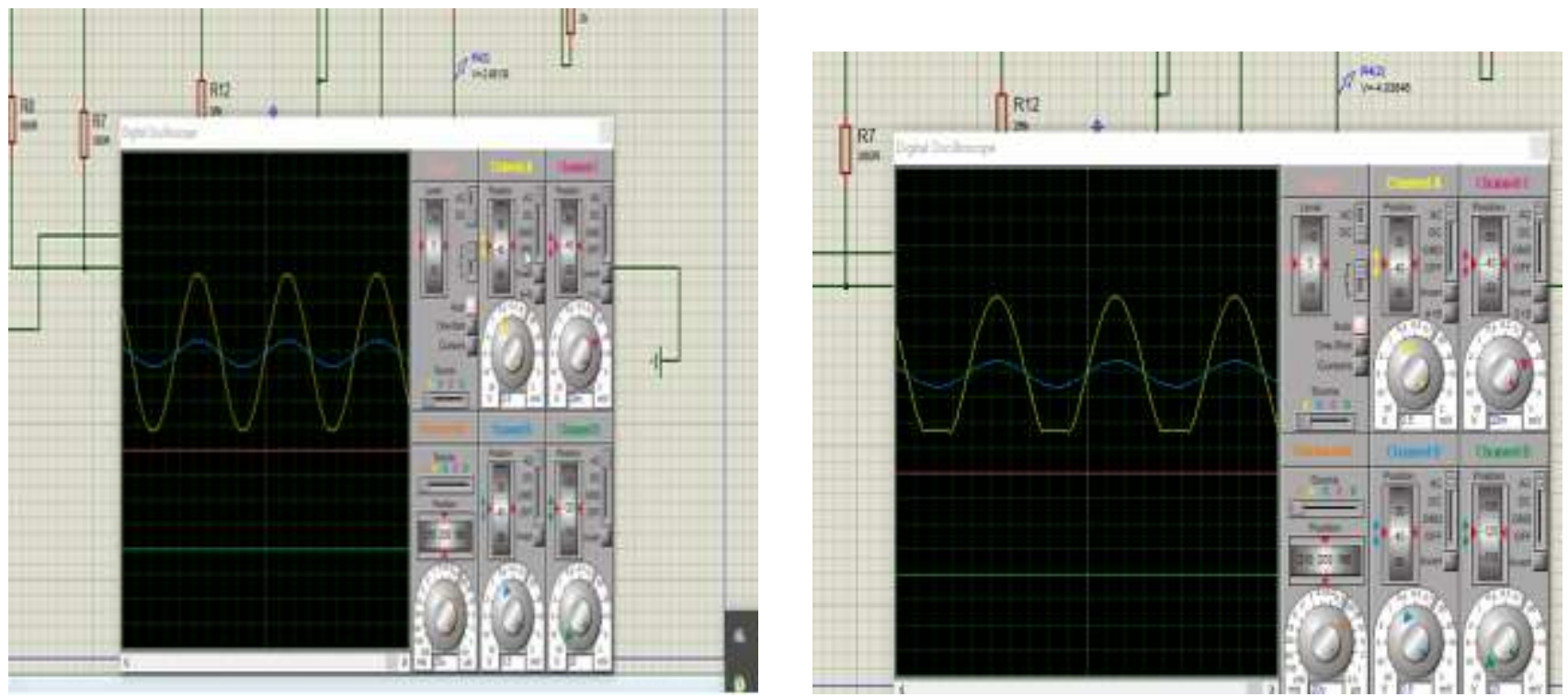

Figure 5.Output Voltage waveform of with negative feedback circuit and without negative feedback circuit 


\subsection{Negative Feedback Amplifier Formula}

From Figure 2 output voltage across $R_{F 1}$, and $\left(R_{F 2},+\mathrm{j} X_{C 2}\right.$, $)$ to give $V_{F}$, so the feedback is

$$
\text { Voltage Feedback }(\mathrm{B})=\frac{\left(R_{F 2},-\mathrm{j} X_{C 2,}\right)}{\left(R_{F 2}, \mathrm{j} X_{C 2},\right)+R_{F 1}}
$$

$$
\text { Assuming }\left(R_{F 2},-\mathrm{j} X_{C 2,}\right)<<R_{F 1}, \quad \text { Voltage Feedback }(\mathrm{B})=\frac{\left(R_{F 2,}-\mathrm{j} X_{C 2,}\right)}{R_{F 1},}
$$

\subsection{Effects of Negative Feedback in Amplifiers Pass Band}

Decibels of Feedback - Effects of Negative Feedback in Amplifiers can be measured in decibels. A statement that $40 \mathrm{~dB}$ of feedback has been applied to an amplifier means that the amplifier gain has been reduced by $40 \mathrm{~dB}$, (that is, by a factor of 100). Consider the typical gain-frequency response of an amplifier, as illustrated in Figure 6. Without negative feedback, the amplifier open-loop $\left(\mathrm{A}_{\mathrm{v}}\right)$ gain falls off to its lower $3 \mathrm{~dB}$ frequency $\left(\mathrm{f}_{1(\mathrm{OL})}\right)$, as illustrated. This is usually due to the impedance of bypass capacitors increasing as the frequency decreases [6].

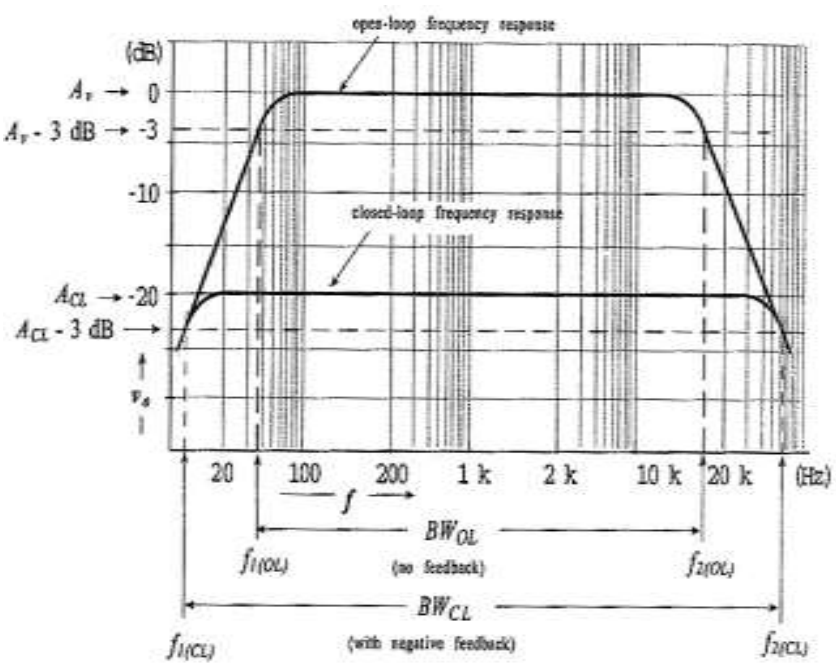

Figure 6.Amplifier frequency response with and without negative feedback, Negative feedback extends the amplifier bandwidth.

Thus $A_{C L}=A_{V}-40 d B=\frac{A_{V}}{100}$

Similarly, the open-loop upper cutoff frequency $\left(\mathrm{f}_{2(\mathrm{OL})}\right)$ is produced by transistor cutoff, by shunting capacitance, or by a combination of both $[3,4,5]$. As discussed earlier, the circuit open-loop bandwidth is,

$B W_{O L}=f_{2(O L)}-f_{1(O L)}$

Now look at the typical frequency response for the same amplifier when Effects of Negative Feedback in Amplifiers is used. The closed-loop gain $\left(\mathrm{A}_{\mathrm{CL}}\right)$ is much smaller than the open-loop gain, and $\mathrm{A}_{\mathrm{CL}}$ does not begin to fall off (at high or low frequencies) until $A_{v}$ (open-loop) falls substantially. Consequently, $\mathrm{f}_{1(\mathrm{CL})}$ is much lower than $\mathrm{f}_{1(\mathrm{OL})}$, and $\mathrm{f}_{2(\mathrm{OL})}$ is much higher than $\mathrm{f}_{2(\mathrm{OL})}$. So, the circuit bandwidth with negative feedback (the closed-loop bandwidth) is much greater than the bandwidth without negative feedback $[3,4,5]$.

$$
B W_{C L}=f_{2(c l)}-f_{1(c l)}
$$

\section{APPLICATION OF NEGATIVE FEEDBACK}

Negative feedback is used in used in the amplifier circuits as they provide the following improvements in the operation of an amplifier. It reduces the stabilize the gain and reduce the distortion, increases the bandwidth. Changing the values of input and output resistances reduces the effects of variation in temperature and supply voltage on the output of the amplifier. Types of negative feedback: depending on the type of sampling and mixing networks, the feedback amplifiers are classified into four categories: voltage series feedback, current series feedback, current shunt feedback, voltage shunt feedback. Application of negative feedback is in the most all the electronic amplifiers, in the regulated power supplies and in amplifiers having a large bandwidth. Feedback network: it is supplied output part to tank circuit to the electronic amplifier [1, 2]. 


\section{COLCLUSION}

By comparing the measurement data and the outcome of Proteus 8 theoretical simulation results indicate that the relationships between close loop and open loop gain are in good agreement with the theory. The overall agreement will satisfy the demand when the circuit is slightly distorted. Negative feedback is presented how to make the difference between hands-on computing and teaching. In this case, human errors should be considered during the actual calculation and the display between different transistors. Using this simulation therefore the circuits conditions can be evaluated particular for complex circuits that can be analyzed by simulation to some extend minimize unnecessary economic expenditure.

\section{REFERENCES}

1. Assaad, R.S. and Silva-Martinez, J. (2009) A Graphical Approach to Teaching Amplifier Design at the Undergraduate Level. IEEE Transactions on Education, 52,39-45. https://doi.org/10.1109/TE.2008.917190

2. Kang, H. (2001) Analog Part in Fundamental of Electronic Technology, 4th Edition, High Education Publishing House, Beijing

3. Leonard S. Bobrow, Fundamentals of Electrical Engineering, Second Edition

4. Thomas L.Floyd, Electronic Devices 9th Edition

5. Yang, Z., Nath, D.N., Zhang, Y.J., Khurgin, B. and Rajan, S. (2015) Common Emitter Current and Voltage Gain in IIINitride Tunneling Hot Electron Transistors. IEEE Electron Device Letters, 36, 436-438. https://doi.org/10.1109/LED.2015.2413934

6. https://www.scribd.com/doc/119790927/Voltage-Series-Feedback-Amplifier

7. https://www.eeeguide.com/two-stage-ce-amplifier-using-series-voltage-negative-feedback/ 